\title{
On Lie algebras of vector fields related to Riemannian foliations
}

\author{
by Tomasz Rybicki (Rzeszów)
}

\begin{abstract}
Riemannian foliations constitute an important type of foliated structures. In this note we prove two theorems connecting the algebraic structure of Lie algebras of foliated vector fields with the smooth structure of a Riemannian foliation.
\end{abstract}

1. Introduction. It is known that the differentiable structure of a smooth manifold $M$ is completely determined by its Lie algebra $\mathfrak{X}(M)$ of all vector fields on $M$ (theorems of L. E. Pursell-M. E. Shanks [11] and I. Amemiya [2]). More recently a very interesting fact was stated by R. P. Filipkiewicz [4]: the group $\operatorname{Diff}^{r}(M)$ of all $C^{r}$ diffeomorphisms of $M$, $1 \leq r \leq \infty$, also determines the $C^{r}$ structure of $M$. The latter result, suggested in part by earlier papers of J. Whittaker and F. Takens, can be regarded as a particularly important contribution to the Erlangen Program of F. Klein.

In this context, also the theorem of Pursell-Shanks and a whole suite of its generalizations may be viewed as an "infinitesimal" contribution to the Program. On the other hand, the interpretation of such theorems in Hamiltonian mechanics is the following. One can say that a generalized phase space is "good" if the Lie algebra of its infinitesimal automorphisms (i.e. the algebra of its symmetries) defines the structure of the phase space itself. Thus numerous geometric structures are proper candidates for being "good" phase spaces. However, this is not always the case even if the algebra of symmetries is infinite-dimensional as some examples show (K. Fukui [5]).

The most general and interesting structure in such studies seems to be a so-called Jacobi manifold or, equivalently, a local Lie algebra over the reals (cf. [7]). In short, it is a nontransitive structure and it generalizes the notions of symplectic and contact manifolds as well as a singular foliation. Therefore

1991 Mathematics Subject Classification: Primary 57R30.

Key words and phrases: Riemannian foliation, Lie algebra, ideal, isomorphism, vector field, generalized manifold, stratification. 
it is an important matter to know whether Pursell-Shanks type theorems hold for foliations (see [2], [6], [12], [13] and also [1], [5], [15]).

In this note we present two results concerning Riemannian foliations. These are foliations with a bundle-like metric (i.e. the leaves remain locally at constant distance) and constitute "a good model for situations drawn from mechanics and physics" (P. Molino [9]).

For simplicity we restrict ourselves to the $C^{\infty}$ smooth category.

2. Statement of main results. Let $(V, \mathcal{F})$ be an arbitrary foliated manifold. By $\mathcal{I}(V, \mathcal{F})$ we denote the Lie algebra of all vector fields tangent to the leaves. A vector field $X$ on $V$ is called foliated if its flow transforms each leaf of $\mathcal{F}$ into a leaf of $\mathcal{F}$. This can also be defined by the property

$$
[X, Y] \in \mathcal{I}(V, \mathcal{F}) \quad \text { for any } Y \in \mathcal{I}(V, \mathcal{F}) \text {. }
$$

Let $\mathfrak{X}(V, \mathcal{F})$ be the Lie algebra of foliated vector fields. Then $\overline{\mathfrak{X}}(V, \mathcal{F})$ stands for the quotient $\mathfrak{X}(V, \mathcal{F}) / \mathcal{I}(V, \mathcal{F})$ and its elements are called transversal vector fields $([8])$. These are sections of the normal bundle $N(V, \mathcal{F})$.

Now let $\left(M_{i}, F_{i}\right), i=1,2$, be nontrivial (i.e. $0<\operatorname{dim} F_{i}<\operatorname{dim} M_{i}$ ) Riemannian foliations with $M_{i}$ compact and connected. Then the leaf closures constitute a new decomposition of $M_{i}$ into imbedded submanifolds, and moreover it is a singular Riemannian foliation denoted by $\bar{F}_{i}$. The leaf space $M_{i} / \bar{F}_{i}$ then admits a Satake manifold structure (cf. [9]).

THEOREM 1. If there exists a Lie algebra isomorphism $\Phi$ of $\overline{\mathfrak{X}}\left(M_{1}, F_{1}\right)$ onto $\overline{\mathfrak{X}}\left(M_{2}, F_{2}\right)$ then there exists a Satake diffeomorphism $\varphi$ of $M_{1} / \bar{F}_{1}$ onto $M_{2} / \bar{F}_{2}$ such that $\varphi_{*}=\Phi$.

THEOREM 2. If there exists a Lie algebra isomorphism $\Phi$ of $\mathfrak{X}\left(M_{1}, F_{1}\right)$ onto $\mathfrak{X}\left(M_{2}, F_{2}\right)$ then there exists a diffeomorphism $\varphi$ of $\left(M_{1}, F_{1}\right)$ onto $\left(M_{2}, F_{2}\right)$ such that $\varphi_{*}=\Phi$.

In the proof of Theorem 2 we shall show that $\Phi\left(\mathcal{I}\left(M_{1}, F_{1}\right)\right)=\mathcal{I}\left(M_{2}, F_{2}\right)$. Hence the following

Corollary. Let $(M, F)$ be a nontrivial Riemannian foliation with $M$ compact and connected. Then the algebraic structure of $\overline{\mathfrak{X}}(M, F)$ determines completely a Satake structure of the leaf space $M / \bar{F}$. Moreover, the algebraic structure of $\mathfrak{X}(M, F)$ defines uniquely both $M / \bar{F}$ and the foliation $(M, F)$ itself.

3. Some properties of Riemannian foliations. We recall briefly some preparatory results on the structure of Riemannian foliations due mainly to Molino ([8], [9]).

Let $M$ be an $n$-dimensional compact and connected manifold equipped with a $q$-codimensional Riemannian foliation $F$. The "Riemannian" prop- 
erty is characterized as follows. Let $(\widetilde{L}(M, F), M, p)$ be the $G L(q)$-principal bundle of transversal frames, i.e. the bundle of linear frames of the normal bundle $N(M, F)$. Then $\widetilde{L}(M, F)$ admits an $O(q)$-reduction, say $(L(M, F), M, p)$. If $(M, F)$ is transversally oriented, then one can assume $L(M, F)$ to be still connected by passing to the $S O(q)$-subbundle.

On the total space $L(M, F)$ one defines a lifted foliation $F_{1}\left(\operatorname{dim} F_{1}=\right.$ $\operatorname{dim} F)$ as follows. Take a submersion $f_{i}: U_{i} \rightarrow f_{i}\left(U_{i}\right), f\left(U_{i}\right) \subset \mathbb{R}^{q}$, defining $F$ on $U_{i}$. Then we have the commutative diagram

$$
\begin{array}{cccc}
p^{-1}\left(U_{i}\right) & \stackrel{f_{i *}}{\longrightarrow} & L\left(f_{i}\left(U_{i}\right)\right) \\
\downarrow & & \downarrow \\
U_{i} & \stackrel{f_{i}}{\longrightarrow} & f_{i}\left(U_{i}\right)
\end{array}
$$

where the vertical arrows are the projections of $O(q)$-bundles. The compatibility conditions are easily checked and $F_{1}$ is defined by the submersions $f_{i *}$. Thus one can say that a leaf of $F_{1}$ is generated by "sliding" a transversal frame along a leaf of $F$. Similarly we define a foliation $\widetilde{F}_{1}$ on $\widetilde{L}(M, F)$.

Now denote by $\widetilde{X}$ the natural lift of $X \in \mathfrak{X}(M, F)$ to $\widetilde{L}(M, F)$. Then it is easily seen that $\widetilde{X} \in \mathfrak{X}\left(\widetilde{L}(M, F), \widetilde{F}_{1}\right)$. Unfortunately, there is no natural lift of $X$ to $L(M, F)$.

However, the following is true.

Proposition 3.1. If $X \in \mathcal{I}(M, F)$ then $\tilde{X} \in \mathcal{I}\left(L(M, F), F_{1}\right)$. Furthermore, if $\overline{\mathcal{I}}(M, F)$ denotes the Lie subalgebra of all foliated vector fields tangent to the foliation $\bar{F}$, then $X \in \overline{\mathcal{I}}(M, F)$ implies that $\widetilde{X} \in \overline{\mathcal{I}}\left(L(M, F), F_{1}\right)$.

P r o of. The first assertion follows immediately from the above diagram. The second is a consequence of the fact that the compact set $L(M, F)$ is an $\bar{F}$-saturated subset of $\widetilde{L}(M, F)$, and that any leaf of $F_{1}$ projects to a leaf of $F$ (so that if $p\left(L_{1}\right)=L$ then $\left.p^{-1}(\bar{L}) \supset \bar{L}_{1}\right)$.

A decisive point is that $\left(L(M, F), F_{1}\right)$ is a transversally parallelizable foliation. In this situation we may apply the Structural Theorem [8]: the closures of the leaves of $F_{1}$ are the fibers of a locally trivial basic fibration

$$
\pi^{T}: L(M, F) \rightarrow W^{T}
$$

where $W^{T}$ is called a basic manifold and its dimension is called the basic dimension of $F_{1}$ (and $F$ ).

Next observe that if $L_{1} \in F_{1}$ and $p\left(L_{1}\right)=L$ then $p\left(\bar{L}_{1}\right)=\bar{L}$. In fact, $p\left(\bar{L}_{1}\right)$ is compact, so it contains $\bar{L}$. By continuity, $\bar{L}_{1} \subset p^{-1}(\bar{L})$. This leads us to the following fundamental fact. The space of leaves of the singular foliation $\bar{F}$ is homeomorphic to the orbit space of an $O(q)$-action on $W^{T}$, i.e. $M / \bar{F}$ is homeomorphic to $W^{T} / O(q)$. In fact, the inverse image $p^{-1}(\bar{L})$ is an $O(q)$-orbit in $W^{T}$. 
Define $\bar{M}=M / \bar{F}, W=W^{T} / O(q)$, and let $h: \bar{M} \rightarrow W$ be the above homeomorphism.

Proposition 3.2. There is a commutative diagram

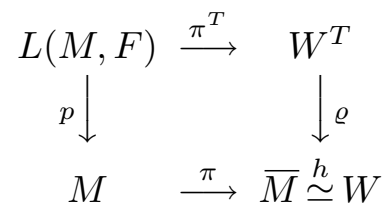

and all arrows are Satake morphisms (with the Satake structure on $W$ being induced by $h)$.

An important feature of $(M, F)$ consists in its stratification. First we take as a stratum, $\Delta_{j}$, the union of leaves of $\bar{F}$ with the same dimension $j$, $j=q, \ldots, n-r$, where $r$ is the basic dimension. Each $\Delta_{j}$ is an imbedded submanifold of $M$. Then we may refine $\left\{\Delta_{j}\right\}$ by taking the substratification $\left\{\Sigma_{\alpha}\right\}$ such that $\Sigma_{\alpha} \subset \Delta_{j}$ and $\Sigma_{\alpha}$ is the union of all leaves in $\Delta_{j}$ with holonomy group of the same fixed cardinality. This possibility follows from the observation that all the leaves of $\bar{F} \mid \Delta_{j}$ have a finite holonomy.

The significance of the resulting stratification is revealed in the following.

Proposition 3.3. The strata $\Sigma_{\alpha}$ are imbedded submanifolds in $M$. The leaf space $\Sigma_{\alpha} / \bar{F} \mid \Sigma_{\alpha}$ is an ordinary manifold.

The proof of the first part appeals to deep properties of Riemannian foliations (see Lemma 5.3 of [9]). The second part is straightforward.

Proposition 3.4. There is a commutative diagram

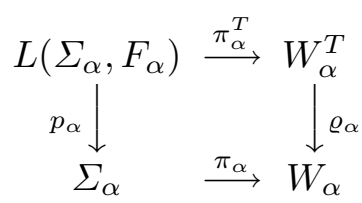

where $F_{\alpha}=F \mid \Sigma_{\alpha}, W_{\alpha}=\Sigma_{\alpha} / \bar{F}_{\alpha}, W_{\alpha}^{T}=\varrho^{-1}\left(W_{\alpha}\right)$, and all maps are restrictions of those in 3.2. Furthermore, all the maps are the projections of locally trivial fibrations.

Proof. The only thing to check is that $\varrho_{\alpha}: W_{\alpha}^{T} \rightarrow W_{\alpha}$ is a locally trivial fibration. This is so because the fibers of $\varrho_{\alpha}$ are compact and the other maps in the diagram are locally trivial fibrations.

Proposition 3.5. Let $X \in \mathfrak{X}(M, F)$. Then $X$ is tangent to each stratum $\Sigma_{\alpha}$.

In fact, the flow of $X$ preserves the leaf closure dimension as well as the cardinality of the holonomy group. 
4. Proof of Theorem 1. The starting point of this section is a theorem due to K. Abe [1] concerning a compact Lie group action on a manifold. Some preparatory definitions are necessary.

Let $G$ be a compact Lie group acting on a connected paracompact manifold $V$. Setting $\bar{V}=V / G$ one has an induced smooth structure on $\bar{V}$ such that $f: \bar{V} \rightarrow \mathbb{R}$ is smooth iff $f \circ \varrho: V \rightarrow \mathbb{R}$ is smooth, where $\varrho$ is the natural projection. As usual, a map $\varphi: \bar{V} \rightarrow \bar{V}^{\prime}, \bar{V}^{\prime}=V^{\prime} / G^{\prime}$, is smooth if $f \circ \varphi \in C^{\infty}(\bar{V})$ for any $f \in C^{\infty}\left(\bar{V}^{\prime}\right)$, and $\varphi$ is a diffeomorphism if both $\varphi$ and $\varphi^{-1}$ are smooth.

Next, proceeding by analogy to the case of a manifold, one defines $D(\bar{V})$ as the Lie algebra of all derivations of $C^{\infty}(\bar{V})$. However, our object of interest is the Lie subalgebra $\mathfrak{X}(\bar{V})$ of compactly supported elements of $D(\bar{V})$ which preserve the stratification of $\bar{V}$ induced by the $G$-action. A theorem of G. W. Schwarz [14] then says that $\mathfrak{X}(\bar{V})$ is the image under $\varrho_{*}$ of $\mathfrak{X}_{G}(V)$, the Lie algebra of all $G$-invariant compactly supported vector fields on $V$. For more details, see [3] and [14].

The proof of the following theorem is long and complicated.

TheOREM 4.1 (Abe). Let $G$ and $G^{\prime}$ be compact Lie groups acting on paracompact and connected manifolds $V$ and $V^{\prime}$, respectively. If there exists a Lie algebra isomorphism $\Phi: \mathfrak{X}(\bar{V}) \rightarrow \mathfrak{X}\left(\bar{V}^{\prime}\right)$ then there exists a strata preserving diffeomorphism $\varphi: \bar{V} \rightarrow \bar{V}^{\prime}$ such that $\varphi_{*}=\Phi$.

Let us return to the Riemannian foliation $(M, F)$. We want to establish that the homeomorphism $h: \bar{M} \rightarrow W$ is actually a Satake diffeomorphism.

Proposition 4.2. If $C^{\infty}(\bar{M})$ is the set of all Satake morphisms of $\bar{M}$ onto $\mathbb{R}$, and $C^{\infty}(W)$ is defined as above $\left(W=W^{T} / O(q)\right)$, then $h^{*} C^{\infty}(W)$ $=C^{\infty}(\bar{M})$. In particular, the smooth structure of the $O(q)$-orbit space $W$ is induced by a Satake manifold structure.

P r o o f. First, making use of Proposition 3.2, we reconstruct the explicit definition of $h^{*}$. Let $f \in C^{\infty}(W)$. Then $f \circ \varrho \circ \pi^{T}$ is a $G$-invariant, $F_{1^{-}}$ foliated function on $L(M, F)$ and there is a unique $g: \bar{M} \rightarrow \mathbb{R}$ such that $g \circ \pi \circ p=f \circ \varrho \circ \pi^{T}$. Of course, $h^{*}(f)=g$.

Next, $f \circ \varrho \circ \pi^{T} \in C^{\infty}(L(M, F))$ so that $g \circ \pi \in C^{\infty}(M)$. Hence, by Lemma 4.3 below, $g \in C^{\infty}(\bar{M})$. On the other hand, by the definition, $\left(h^{-1}\right)^{*}(f) \in$ $C^{\infty}(W)$ if $f \in C^{\infty}(\bar{M})$.

LEMma 4.3. $f \in C^{\infty}(\bar{M})$ iff $f \circ \pi \in C^{\infty}(M)$.

This follows easily from the fact that the Satake morphism $\pi$ is (locally) induced by a submersion. We leave the details to the reader.

Now we are in a position to conclude the proof of Theorem 1 . In view of Proposition 4.2, the proof reduces to an application of Theorem 4.1 if we 
show that $\overline{\mathfrak{X}}(M, F)$ is isomorphic to $\mathfrak{X}(W)$, the latter being defined as at the beginning of this section.

It follows from the commutative diagram

$$
\begin{array}{ccc}
p^{-1} N(M, F) & \stackrel{p_{*}}{\longrightarrow} & N(M, F) \\
\downarrow & & \downarrow \\
L(M, F) & \stackrel{p}{\longrightarrow} & M
\end{array}
$$

that $\overline{\mathfrak{X}}(M, F)$, the totality of global sections of $N(M, F)$, is pulled back isomorphically to the set of $O(q)$-invariant sections of $p^{-1} N(M, F)$. Equivalently, this is the Lie algebra of all $O(q)$-invariant $F_{1}$-transversal vector fields on $L(M, F)$ modulo ker $p_{*}$. The latter in turn projects isomorphically under $\pi_{*}^{T}$ to the Lie algebra of all $O(q)$-invariant vector fields on $W^{T}$ modulo ker $\varrho_{*}$, $\mathfrak{X}_{O(q)}\left(W^{T}\right) / \operatorname{ker} \varrho_{*}$. Finally, by the theorem of Schwarz, $\mathfrak{X}_{O(q)}\left(W^{T}\right) / \operatorname{ker} \varrho_{*}$ is isomorphic to $\mathfrak{X}(W)$.

5. Proof of Theorem 2. We shall make use of the diagram in Proposition 3.4. Let us fix a stratum $\Sigma_{\alpha}$ from the stratification defined in $\S 3$. Define a homomorphism

$$
\tilde{\pi}_{\alpha}: \mathfrak{X}(M, F) \rightarrow \mathfrak{X}\left(W_{\alpha}\right)
$$

such that $\pi_{\alpha *}\left(X \mid \Sigma_{\alpha}\right)=\tilde{\pi}_{\alpha}(X)$. Let $\mathcal{A}_{\alpha}$ be the image of $\mathfrak{X}(M, F)$ under $\tilde{\pi}_{\alpha}$.

The proof of the next proposition follows a reasoning due to M. Pierrot [10].

Proposition 5.1. $\mathfrak{X}_{\mathrm{c}}\left(W_{\alpha}\right) \subset \mathcal{A}_{\alpha}$, where $\mathfrak{X}_{\mathrm{c}}\left(W_{\alpha}\right)$ is the Lie algebra of compactly supported vector fields on the manifold $W_{\alpha}$.

P r o of. Let $X \in \mathfrak{X}_{\mathrm{c}}\left(W_{\alpha}\right)$. Bearing in mind that $\varrho_{\alpha}: W_{\alpha}^{T} \rightarrow W_{\alpha}$ is a locally trivial fibration, the compactly supported $X$ lifts to some $X^{T}$ on $W_{\alpha}^{T}$, $X^{T}$ being also with compact support. This vector field, in turn, extends from the imbedded submanifold $W_{\alpha}^{T}$ to the manifold $W^{T}$; the resulting vector field is still denoted by $X^{T}$. Now $\left(L(M, F), F_{1}\right)$ is transversally parallelizable, so that one can lift $X^{T}$ to a foliated vector field $Y^{T}$ on $L(M, F)$. Next we transform $Y^{T}$ to an $S O(q)$-invariant and still $F_{1}$-foliated vector field $\widetilde{Y}^{T}$, namely

$$
\widetilde{Y}^{T}=\int_{S O(q)}\left(R_{g}^{*} Y^{T}\right) d \mu,
$$

where $\mu$ is the normalized Haar measure on $S O(q)$. It is evident that $\varrho_{\alpha *} \pi_{*}^{T}\left(Y^{T}\right)=X$, so that $\tilde{\pi}_{\alpha}(Y)=X$ where $Y=p_{*}\left(\widetilde{Y}^{T}\right)$.

This completes the proof.

Now we try to describe some maximal ideals in $\mathfrak{X}(M, F)$ by means of the homomorphisms $\widetilde{\pi}_{\alpha}$. We start with some generalities. 
Let $V$ be a manifold and $p \in V$. We denote by $S_{p}=S_{p}(\mathfrak{X}(V))$ the Lie subalgebra in $\mathfrak{X}(V)$ formed by all vector fields vanishing at $p$. If $\mathcal{A}$ is a Lie subalgebra of $\mathfrak{X}(V)$, then we set $S_{p}(\mathcal{A})=\mathcal{A} \cap S_{p}$. Next

$$
\begin{aligned}
m_{p}=m_{p}(\mathfrak{X}(V))=\left\{X \in \mathfrak{X}(V):\left[Y_{1}, \ldots,\left[Y_{r}, X\right] \ldots\right]\right. & \in S_{p}, \\
\forall r & \left.\geq 0 \forall Y_{1}, \ldots, Y_{r} \in \mathfrak{X}(V)\right\},
\end{aligned}
$$

and more generally for any Lie subalgebra $\mathcal{A}$ we set

$$
m_{p}(\mathcal{A})=\left\{X \in \mathcal{A}:\left[Y_{1}, \ldots,\left[Y_{r}, X\right] \ldots\right] \in S_{p}, \forall r \geq 0 \forall Y_{1}, \ldots, Y_{r} \in \mathcal{A}\right\} .
$$

Then $m_{p}$ and $m_{p}(\mathcal{A})$ are ideals of $\mathfrak{X}(V)$ and $\mathcal{A}$, respectively. Observe that $\mathcal{A} \cap m_{p} \subset m_{p}(\mathcal{A})$, but equality does not hold (e.g. for $\mathcal{A}=\mathcal{I}(V, \mathcal{F}), \mathcal{F}$ being a foliation with $\operatorname{dim}>0$ ).

The proof of the following is in [11], [12].

Proposition 5.2. Let $(V, \mathcal{F})$ be an arbitrary foliation with $\operatorname{dim}>0$. The ideals $m_{p}(\mathcal{I}(V, \mathcal{F})), p \in V$, are the unique maximal ideals in $\mathcal{I}_{\mathrm{c}}(V, \mathcal{F})$. In particular, $m_{p}\left(\mathfrak{X}_{\mathrm{c}}(V)\right)$ are the unique maximal ideals in $\mathfrak{X}_{\mathrm{c}}(V)$.

As usual "c" indicates compactly supported subalgebras.

Lemma 5.3. Let $\operatorname{dim} W_{\alpha}>0$ and $x \in W_{\alpha}$. Then $m_{x}\left(\mathcal{A}_{\alpha}\right)$ is a maximal ideal in $\mathcal{A}_{\alpha}$.

P r o of. We make use of Proposition 5.1. Let $I$ be an ideal in $\mathcal{A}_{\alpha}$ such that $m_{x}\left(\mathcal{A}_{\alpha}\right) \varsubsetneqq I$ for some $x$. Then a standard argument shows that $\mathfrak{X}_{\mathrm{c}}\left(W_{\alpha}\right) \subset I$. Furthermore, if $X \in \mathcal{A}_{\alpha}$ then clearly $X=X_{1}+X_{2}$ with $X_{1} \in \mathfrak{X}_{\mathrm{c}}\left(W_{\alpha}\right)$, $X_{2} \in m_{x}\left(\mathfrak{X}\left(W_{\alpha}\right)\right)$. Hence $X_{2} \in \mathcal{A}_{\alpha}$. But $\mathcal{A}_{\alpha} \cap m_{x} \subset m_{x}\left(\mathcal{A}_{\alpha}\right)$ so that $X_{2} \in I$ and $X \in I$. Thus $I=\mathcal{A}_{\alpha}$.

R e m ark. Simple examples (modifying an example in [13]) show that the $m_{x}\left(\mathcal{A}_{\alpha}\right)$ need not be unique maximal ideals.

We introduce the following notation: $M=\Sigma_{0} \cup \Sigma_{1}$ where $\Sigma_{0}$ is the union of all singular strata, i.e. the strata $\Sigma_{\alpha}$ with $\operatorname{dim} W_{\alpha}=0$, and $\Sigma_{1}$ the union of all regular strata.

Definition. Let $L \in \bar{F}, L \subset \Sigma_{1}$. Set $\mu_{L}=\tilde{\pi}_{\alpha}^{-1}\left(m_{x}\right)$ where $\pi_{\alpha}(L)=\{x\}$.

Then by Lemma $5.3, \mu_{L}$ is a maximal ideal in $\mathfrak{X}(M, F)$.

Now our goal is to get rid of maximal ideals which do not contain $\overline{\mathcal{I}}(M, F)$. The following observations show that these are ideals connected with singular strata.

Proposition 5.4. Let $I$ be a maximal ideal of $\mathfrak{X}(M, F)$. If $I \neq$ $m_{p}(\mathfrak{X}(M, F))$ for any $p \in \Sigma_{0}$ then $\mathcal{I}(M, F) \subset I$.

Proof. First observe that $I \not \subset m_{p}(\mathfrak{X}(M, F))$ for any $p \in \Sigma_{1}$. In fact, if $I \subset m_{p}(\mathfrak{X}(M, F))$ for $p \in L \in \bar{F}$ then $I \varsubsetneqq \mu_{L}$, contrary to the maximality 
of $I$. Thus, in view of the definition of $m_{p}(\mathfrak{X}(M, F))$, for any $p \in M$ there is an $X \in I$ such that $X(p) \neq 0$.

Fix for a moment $p \in M$ and $X \in I$ with $X(p) \neq 0$. One can take a distinguished chart $\left(U, x_{1}, \ldots, x_{n-q}, y_{1}, \ldots, y_{q}\right)$ at $p$ (i.e. $x_{i}(p)=0, y_{j}(p)=0$ and for $y_{1}, \ldots, y_{q}$ fixed, $x_{1}, \ldots, x_{n-q}$ are local coordinates of a leaf) such that either

(a) $X=\partial / \partial x_{1}+\sum_{j} g_{j}\left(y_{1}, \ldots, y_{q}\right) \partial / \partial y_{j}$ with $g(0)=0$ (if $X$ is tangent at $p$ ), or

(b) $X=\partial / \partial y_{1}$ (if $X$ is not tangent at $p$ ),

on a neighborhood of $p$. This follows from the fact that any foliated vector field can be locally written in a distinguished chart in the form

$$
\sum f_{i}(x, y) \partial / \partial x_{i}+\sum g_{j}(y) \partial / \partial y_{j}
$$

where $x=\left(x_{1}, \ldots, x_{n-q}\right), y=\left(y_{1}, \ldots, y_{q}\right)$, and from the possibility of a special choice of a distinguished chart (see e.g. [5], [6]).

We wish to modify the form of $X$ in the case (a). As $\mathcal{I}(M, F)$ is a $C^{\infty}(M)$-module, a standard reasoning gives the existence of $Z \in \mathcal{I}(M, F)$ such that $Z=x_{1} \partial / \partial x_{1}$ on a neighborhood of $p$. Define $Y=[X, Z]$. Then $Y=\left[\partial / \partial x_{1}+\sum_{j} g_{j}(y) \partial / \partial y_{j}, x_{1} \partial / \partial x_{1}\right]=\partial / \partial x_{1}$ on a neighborhood of $p$. Thus we may assume that for any $p \in M$ there exists $Y \in I$ such that $Y=\partial / \partial x_{1}$ or $\partial / \partial y_{1}$ in some distinguished chart at $p$. Let $M=\bigcup_{i=1}^{r} U_{i}$ be a finite open cover ( $M$ is compact) consisting of domains of charts satisfying the above property (i.e. an extension to $M$ of $\partial / \partial x_{1}$ or $\partial / \partial y_{1}$ belongs to $I$ ).

We wish to show that $\mathcal{I}(M, F) \subset I$. For $X \in I$ we have a decomposition $X=X_{1}+\ldots+X_{r}$ with $X_{i} \in \mathcal{I}(M, F)$, supp $X_{i} \subset U_{i}$ (it suffices to multiply $X$ by a partition of unity subordinate to $\left\{U_{i}\right\}$ ). Hence we may assume that $\operatorname{supp} X \subset U, U$ being a distinguished chart domain, and that an extension $Y$ of $\partial / \partial x_{1}$ belongs to $I$ (for $\partial / \partial y_{1}$ the proof is the same). Let $X=\sum f_{i}(x, y) \partial / \partial x_{i}$ on $U$. Choose a smooth function $\varphi$ such that $\operatorname{supp} \varphi \subset U$ and $\varphi=1$ on a neighborhood of $\operatorname{supp} X$. Next let a smooth function $\psi$ satisfy $\operatorname{supp} \psi \subset U, \psi=1$ on a neighborhood of $\operatorname{supp} \varphi$. Then for $i=1, \ldots, n-q$ we have

$$
\left[Y,\left(\psi \int_{-\infty}^{x_{1}} \varphi d x_{1}\right) \partial / \partial x_{i}\right]=Z+\varphi \partial / \partial x_{i}
$$

where $\operatorname{supp} Z \subset M \backslash \operatorname{supp} \varphi$, and

$$
\begin{aligned}
{\left[Z+\varphi \partial / \partial x_{i},\left(\varphi \int_{-\infty}^{x_{i}} f_{i} d x_{i}\right) \partial / \partial x_{i}\right] } & =\left[\varphi \partial / \partial x_{i},\left(\varphi \int_{-\infty}^{x_{i}} f_{i} d x_{i}\right) \partial / \partial x_{i}\right] \\
& =f_{i} \partial / \partial x_{i} .
\end{aligned}
$$


Therefore $f_{i} \partial / \partial x_{i} \in I$ and $X \in I$, which completes the proof.

Corollary 5.5. The ideal $m_{p}(\mathfrak{X}(M, F)), p \in \Sigma_{0}$, is either maximal, or contained in a maximal finite-codimensional ideal I such that $\mathcal{I}(M, F) \subset$ $I \subset \overline{\mathcal{I}}(M, F)$.

In fact, if $p \in L \in F, L$ closed, then by the above reasoning $m_{p}(\mathfrak{X}(M, F))$ is maximal. On the other hand, if $p \in L \neq \bar{L}$ then $I=\{X \in \mathfrak{X}(M, F): X$ is tangent to $F$ on $\bar{L}\}$ satisfies the assertion.

Definition. A maximal ideal $I$ of $\mathfrak{X}(M, F)$ is said to be distinguished if $\overline{\mathcal{I}}(M, F) \subset I$. E.g. the $\mu_{L}$ are distinguished ideals.

We are going to find an algebraic property which characterizes the distinguished ideals. For any Lie subalgebra $\mathcal{A}$ of $\mathfrak{X}(M, F)$ we denote by $\mathcal{A}^{*}$ the set of all maximal ideals of $\mathcal{A}$. Our characterization is based on the topological properties of $\mathcal{A}^{*}$.

We introduce the Stone topology on $\mathcal{A}^{*}$ in the following way. Let $\Omega$ be a subset of $\mathcal{A}^{*}$. Then the closure of $\Omega$ is defined by

$$
\bar{\Omega}=\left\{\mu \in \mathcal{A}^{*}: \bigcap\{\omega \in \Omega\} \subset \mu\right\} .
$$

In particular, $\bar{\emptyset}=\emptyset$.

Remark. Let $(V, \mathcal{F})$ be any foliated manifold with $\operatorname{dim}>0$. It is an easy consequence from Proposition 5.2 that the mapping $V \ni p \rightarrow$ $m_{p}\left(\mathcal{I}_{\mathrm{c}}(V, \mathcal{F})\right) \in \mathcal{I}_{\mathrm{c}}(V, \mathcal{F})^{*}$ is a homeomorphism. Specifically, $V$ and $\mathfrak{X}_{\mathrm{c}}(V)^{*}$ are homeomorphic.

Now we consider the decomposition of $\mathfrak{X}(M, F)^{*}$ into connected components in the Stone topology. We wish to show that such a component either contains distinguished ideals only, or contains no distinguished ideals at all. This follows from

Proposition 5.6. Let $\mathcal{D}$ (respectively, $\mathcal{N}$ ) denote the set of all distinguished (respectively, nondistinguished) ideals of $\mathfrak{X}(M, F)$. Then $\overline{\mathcal{D}} \cap \mathcal{N}=\emptyset$ and $\mathcal{D} \cap \overline{\mathcal{N}}=\emptyset$.

Proof. If $I \in \mathfrak{X}(M, F)^{*}$ and $\bigcap \mathcal{D} \subset I$ then $\mathcal{I}(M, F) \subset I$ and $I$ has to be distinguished. Now let $I \in \mathfrak{X}(M, F)^{*}$ be such that $\bigcap \mathcal{N} \subset I$, and $I \in \mathcal{D}$. Take a foliated vector field $X \notin I$. The $\bar{F}$-orthogonal part $X^{\prime}$ of $X$ satisfies $X^{\prime} \notin I$ since $\mathcal{I}(M, F) \subset I$. Now, by definition, for any $p \in \Sigma_{0}$,

$$
\begin{aligned}
X^{\prime} \in m_{p}(\mathfrak{X}(M, F)) \Leftrightarrow\left[Y_{1}, \ldots,\left[Y_{r}, X\right] \ldots\right] & \in S_{p}(\mathfrak{X}(M, F)), \\
\forall r & \geq 0 \forall Y_{1}, \ldots, Y_{r} \in \mathfrak{X}(M, F) .
\end{aligned}
$$

This condition is indeed satisfied as the tangent part of $\left[Y_{i}, \ldots,\left[Y_{r}, X\right] \ldots\right]$ vanishes, and the orthogonal part must be 0 on singular strata. Thus $X^{\prime} \in$ $\bigcap \mathcal{N}$ by Corollary 5.5 and $X^{\prime} \notin I$, a contradiction. 
Now we are in a position to give the required characterization of distinguished ideals. We introduce the following notation. Letting $\mathcal{A}$ denote a Lie subalgebra of $\mathfrak{X}(M, F), \mathcal{A}^{\#}$ stands for the set of all ideals of $\mathcal{A}$ which are maximal in the set of all infinite-codimensional ideals of $\mathcal{A}$.

For any $L \in \bar{F}$ consider $\nu_{L}=\{X \in \overline{\mathcal{I}}(M, F): X$ is tangent to $F$ on $\bar{L}\}$, which is an ideal in $\overline{\mathcal{I}}(M, F)$. Then $\nu_{L}$ is finite-codimensional in $\overline{\mathcal{I}}(M, F)$, since the foliation $F \mid L$ has all its leaves dense. By contrast, $m_{p}(\mathcal{I}(M, F))$ is infinite-codimensional in $\mathcal{I}(M, F)$ as well as $\mu_{L}$ is infinite-codimensional in $\mathfrak{X}(M, F)$.

Proposition 5.7. $\mu \in \mathfrak{X}(M, F)^{*}$ is distinguished if and only if $\mu$ belongs to an element of a unique minimal family $\mathcal{R}$ of connected components of $\mathfrak{X}(M, F)^{*}$ characterized by the equality

$$
\bigcap\left\{\varrho \in(\bigcap \widetilde{\mathcal{R}})^{\#}\right\}=0
$$

where $\widetilde{\mathcal{R}}=\bigcup \mathcal{R}$.

P r o of. Suppose $\widetilde{\mathcal{R}}=\mathcal{D}$. Then $\bigcap \widetilde{\mathcal{R}}=\overline{\mathcal{I}}(M, F)$. Next observe the following implication for any ideal $I$ of $\overline{\mathcal{I}}(M, F)$ :

$$
m_{p}(\overline{\mathcal{I}}(M, F)) \varsubsetneqq I \Rightarrow \nu_{L} \subset I .
$$

Hence $m_{p}(\overline{\mathcal{I}}(M, F)) \in(\overline{\mathcal{I}}(M, F))^{\#}$ for any $p$. This gives

$$
\bigcap\left\{\varrho \in(\overline{\mathcal{I}}(M, F))^{\#}\right\}=0 \text {. }
$$

Suppose now that $\mathcal{R}$ does not contain a distinguished component, say $\mathcal{C}$. It follows easily from the definition of the topology that $\left\{\mu_{L}\right\}$ is dense in $\mathcal{D}$. Hence $\mu_{L} \in \mathcal{C}$ for some $L$. If $L \subset \Sigma_{\alpha}$ then again by the definition of the topology, $\mu_{L^{\prime}} \in \mathcal{C}$ for every $L^{\prime} \subset \Sigma_{\alpha}$. Then bearing in mind the definition of strata, $\tilde{\pi}_{\alpha}^{-1}\left(\mathfrak{X}_{\mathrm{c}}\left(W_{\alpha}\right)\right)\left|\Sigma_{\alpha} \subset \bigcap \widetilde{\mathcal{R}}\right| \Sigma_{\alpha}$ (this makes sense in view of Proposition 3.5). Now let $J \in(\bigcap \widetilde{\mathcal{R}})^{\#}$. If $\mathcal{I}(M, F)\left|\Sigma_{\alpha} \not \subset J\right| \Sigma_{\alpha}$ then

$$
J\left|\Sigma_{\alpha} \subset m_{p}(\bigcap \widetilde{\mathcal{R}})\right| \Sigma_{\alpha} \varsubsetneqq \mu_{L} \cap \bigcap \widetilde{\mathcal{R}} \mid \Sigma_{\alpha},
$$

which contradicts the definition of $\#$ as $\mu_{L}$ is infinite-codimensional in $\bigcap \widetilde{\mathcal{R}}$. Consequently, $\mathcal{I}(M, F)\left|\Sigma_{\alpha} \subset J\right| \Sigma_{\alpha}$ and $\bigcap\left\{\varrho \in(\bigcap \widetilde{\mathcal{R}})^{\#}\right\}$ is nonzero. This and the minimality of $\mathcal{R}$ imply the proposition.

Corollary 5.8. $\overline{\mathcal{I}}(M, F)$ is the intersection of all distinguished ideals.

Corollary 5.9. $\mathcal{I}(M, F)$ is the intersection, $\mathcal{M}$, of all ideals of $\overline{\mathcal{I}}(M, F)$ with finite codimension.

Proof. By an argument from the proof of Proposition 5.4 any ideal $I$ of $\overline{\mathcal{I}}(M, F)$ which does not contain $\mathcal{I}(M, F)$ must be contained in some 
$m_{p}(\overline{\mathcal{I}}(M, F))$. This means that $\mathcal{I}(M, F) \subset \mathcal{M}$. Conversely, $\nu_{L}$ is finitecodimensional for all $L \in \bar{F}$ and $\mathcal{I}(M, F)=\bigcap \nu_{L}$. This completes the proof.

COROLLARY 5.10. Under the assumptions formulated before the statement of Theorem $2, \Phi\left(\mathcal{I}\left(M_{1}, F_{1}\right)\right)=\mathcal{I}\left(M_{2}, F_{2}\right)$.

This follows from Corollaries 5.8 and 5.9.

Now to end the proof of Theorem 2 we just apply the Amemiya's theorem, which for our purposes can be reformulated as follows (cf. [12]):

THEOREM 5.11 [2]. If $\Phi$ is a Lie algebra isomorphism of $\mathfrak{X}\left(M_{1}, F_{1}\right)$ onto $\mathfrak{X}\left(M_{2}, F_{2}\right)$ such that $\Phi\left(\mathcal{I}\left(M_{1}, F_{1}\right)\right)=\mathcal{I}\left(M_{2}, F_{2}\right)$, then there is a foliation preserving diffeomorphism $\varphi$ of $M_{1}$ onto $M_{2}$ such that $\varphi_{*}=\Phi$ on $\mathfrak{X}\left(M_{1}, F_{1}\right)$.

Acknowledgements. The author is greatly indebted to the referee for pointing out a gap in the proof of Theorem 2 and for other comments.

Added in proof (April 1993). Recently Janusz Grabowski sent me his paper (Lie algebras of vector fields and generalized foliations, preprint) where he proved a much stronger version of my Theorem 2. Namely, the theorem holds true for any generalized foliation, also in the real-analytic and holomorphic categories. The proof is based on algebraic properties of modular Lie algebras of vector fields.

\section{References}

[1] K. Abe, Pursell-Shanks type theorem for orbit spaces of $G$-manifolds, Publ. RIMS Kyoto Univ. 18 (1982), 685-702.

[2] I. Amemiya, Lie algebra of vector fields and complex structure, J. Math. Soc. Japan 27 (1975), 545-549.

[3] M. Davis, Smooth G-manifolds as collections of fiber bundles, Pacific J. Math. 77 (1978), 315-363.

[4] R. P. Filipkiewicz, Isomorphisms between diffeomorphism groups, Ergodic Theory Dynamical Systems 2 (1982), 159-171.

[5] K. Fukui, Pursell-Shanks type theorem for free G-manifolds, Publ. RIMS Kyoto Univ. 17 (1981), 249-265.

[6] K. Fukui and N. Tomita, Lie algebra of foliation preserving vector fields, J. Math. Kyoto Univ. 22 (1983), 685-699.

[7] F. Guedira et A. Lichnerowicz, Géométrie des algèbres de Lie locales de Kirillov, J. Math. Pures Appl. 63 (1984), 407-484.

[8] P. Molino, Géométrie globale des feuilletages riemanniens, Nederl. Akad. Wetensch. Proc. 85 (1982), 45-76.

[9] —, Riemannian Foliations, Progr. Math. 73, Birkhäuser, 1988.

[10] M. Pierrot, Orbites des champs feuilletés pour un feuilletage riemannien sur une variété compacte, C. R. Acad. Sci. Paris 301 (1985), 443-445.

[11] L. E. Pursell and M. E. Shanks, The Lie algebra of a smooth manifold, Proc. Amer. Math. Soc. 5 (1954), 468-472.

[12] T. Rybicki, On the Lie algebra of a transversally complete foliation, Publ. Sec. Mat. Univ. Autònoma Barcelona 31 (1987), 5-16. 
[13] T. Rybicki, Lie algebras of vector fields and codimension one foliations, Publ. Mat. 34 (1990), 311-321.

[14] G. W. Schwarz, Lifting smooth homotopies of orbit spaces, Publ. IHES 51 (1980), 37-135.

[15] R. A. Wolak, Maximal subalgebras in the algebra of foliated vector fields of a Riemannian foliation, Comment. Math. Helv. 64 (1989), 536-541.

INSTITUTE OF MATHEMATICS

PEDAGOGICAL UNIVERSITY

REJTANA 16A

35-310 RZESZÓW, POLAND

Reçu par la Rédaction le 5.10.1990

Révisé le 20.5.1992 et 14.9.1992 